\title{
TU/e emonownen

\section{Statistical properties of turbulence in the presence of a smart small-scale control}

\section{Citation for published version (APA):}

Buzzicotti, M., Biferale, L., \& Toschi, F. (2020). Statistical properties of turbulence in the presence of a smart small-scale control. Physical Review Letters, 124(8), [084504]. https://doi.org/10.1103/PhysRevLett.124.084504

DOI:

10.1103/PhysRevLett.124.084504

Document status and date:

Published: 28/02/2020

\section{Document Version:}

Publisher's PDF, also known as Version of Record (includes final page, issue and volume numbers)

\section{Please check the document version of this publication:}

- A submitted manuscript is the version of the article upon submission and before peer-review. There can be important differences between the submitted version and the official published version of record. People interested in the research are advised to contact the author for the final version of the publication, or visit the $\mathrm{DOI}$ to the publisher's website.

- The final author version and the galley proof are versions of the publication after peer review.

- The final published version features the final layout of the paper including the volume, issue and page numbers.

Link to publication

\section{General rights}

Copyright and moral rights for the publications made accessible in the public portal are retained by the authors and/or other copyright owners and it is a condition of accessing publications that users recognise and abide by the legal requirements associated with these rights.

- Users may download and print one copy of any publication from the public portal for the purpose of private study or research.

- You may not further distribute the material or use it for any profit-making activity or commercial gain

- You may freely distribute the URL identifying the publication in the public portal.

If the publication is distributed under the terms of Article 25fa of the Dutch Copyright Act, indicated by the "Taverne" license above, please follow below link for the End User Agreement:

www.tue.nl/taverne

Take down policy

If you believe that this document breaches copyright please contact us at:

openaccess@tue.nl

providing details and we will investigate your claim. 


\title{
Statistical Properties of Turbulence in the Presence of a Smart Small-Scale Control
}

\author{
Michele Buzzicotti, ${ }^{1}$ Luca Biferale, ${ }^{1}$ and Federico Toschi ${ }^{2, *}$ \\ ${ }^{1}$ Department of Physics and INFN, University of Rome "Tor Vergata," Via della Ricerca Scientifica 1, 00133 Rome, Italy \\ ${ }^{2}$ Department of Applied Physics, Eindhoven University of Technology, $5600 \mathrm{MB}$ Eindhoven, \\ The Netherlands and Istituto per le Applicazioni del Calcolo, Consiglio Nazionale delle Ricerche, 00185 Rome, Italy
}

(Received 24 May 2019; accepted 6 January 2020; published 28 February 2020)

\begin{abstract}
By means of high-resolution numerical simulations, we compare the statistical properties of homogeneous and isotropic turbulence to those of the Navier-Stokes equation where small-scale vortex filaments are strongly depleted, thanks to a nonlinear extra viscosity acting preferentially on high vorticity regions. We show that the presence of such smart small-scale drag can strongly reduce intermittency and non-Gaussian fluctuations. Our results pave the way towards a deeper understanding on the fundamental role of degrees of freedom in turbulence as well as on the impact of (pseudo)coherent structures on the statistical small-scale properties. Our work can be seen as a first attempt to develop smart-Lagrangian forcing or drag mechanisms to control turbulence.
\end{abstract}

DOI: 10.1103/PhysRevLett.124.084504

Introduction.-Fluid dynamics turbulence is characterized by intermittent and non-Gaussian fluctuations distributed over a wide range of space and timescales [1-6]. In the limit of infinite Reynolds numbers, Re, the number of dynamical degrees of freedom tends towards infinity, $\#_{\text {dof }} \sim \mathrm{Re}^{9 / 4}$, where $\operatorname{Re}=U_{0} L_{0} / \nu$ with $\nu$ being the viscosity, $U_{0}$ and $L_{0}$ being the typical velocity and large scale in the flow, respectively. Are all these degrees of freedom equally relevant for the dynamics? Do extreme events depend only on some large-scale flow realizations? Can we selectively control some degrees of freedom by applying an active forcing and/or drag? These are key questions that we start to answer by using high resolution numerical studies of the three dimensional Navier-Stokes equations. The long term goal is twofold. First, we are interested to have a new numerical tool to ask novel questions concerning the statistical and topological properties of specific flow structures. Second, we aim to develop useful control strategies to suggest forcing protocols that may be implemented in laboratory experiments, where the flow can be seeded with millions of passive or active particles, preferentially tracking special flow regions [7-14]. For example, we nowadays know how to actively control spinning properties of small magnetic particles $[15,16]$, how to blow up small bubbles by sound emissions [17-19], and/or how to assemble micrometric objects with a selfadaptive shape depending on the flow rheological properties [20]. Recent developments in 3D printing and microengineering technologies promise that new tools will be available in the next few years for fluid control or fluid measurements in the laboratory. We believe that these new tools could be capable to do, in a "smart" way, what passive polymers already do in controlling drag and flow correlations [21-23]. In this Letter, we perform a first attempt to modify or control fluid turbulence by adding a small-scale forcing only on intense vorticity regions. We start from the case where the forcing is always detrimental, i.e., removes energy. The idea is to have a numerical experiment mimicking the effects of small particles that preferentially track high vorticity regions (i.e., light bubbles) and that can be activated such as to spin or blowup and increase the drag locally. This is only one potential protocol over a wide and broad range of other applications to many others flow conditions at high and low Reynolds numbers.

Method.-We consider the Navier Stokes equations (NSE) for an incompressible flow, subjected to two different types of forcing mechanisms:

$$
\partial_{t} \boldsymbol{u}+\boldsymbol{u} \cdot \boldsymbol{\nabla} \boldsymbol{u}=-\boldsymbol{\nabla} P+\nu \Delta \boldsymbol{u}+\boldsymbol{F}-\boldsymbol{f}_{c},
$$

where $\mathbf{F}$ is a standard large-scale stirring mechanism while $\boldsymbol{f}_{c}$ is a second forcing which acts-in our implementationas a control term on the small-scale dynamics. In particular, in this Letter, we will only consider an external smart drag, proportional to the velocity $\boldsymbol{f}_{c}(\boldsymbol{x}, t)=c(\boldsymbol{x}, t) \boldsymbol{u}(\boldsymbol{x}, t)$ and acting such as to preferentially depleting only those regions where vorticity is important

$$
c(\boldsymbol{x}, t)=\beta\left(\frac{\tanh \left\{\left[\omega(\boldsymbol{x}, t)-\omega_{p}\right]\right\}+1}{2}\right),
$$

where $\omega(\boldsymbol{x}, t)=|\boldsymbol{\nabla} \times \boldsymbol{u}|$ is the vorticity intensity, $\omega_{p}$ is one threshold above which the control term is strongly active and $\beta$ is an overall rescaling factor of the control amplitude, hence $\beta=0$ would correspond to the usual NSE without control. From its definition it is possible to see that $\boldsymbol{f}_{c}(\boldsymbol{x}, t)$ is always close to zero except inside structures dominated by the intense vortex filaments, where the tanh 
becomes positive and equal to 1 . The region where $\boldsymbol{f}_{c}(\boldsymbol{x}, t)$ is acting and can be tuned by changing the threshold $\omega_{p}$, whose value has been fixed as a percentage of the maximum vorticity, $\omega_{\max }$, measured in the stationary state of a simulation without the control term, hence:

$$
\omega_{p}=p \omega_{\max },
$$

with $0<p \leq 1$. In the transition region around the isoline where $\omega(\boldsymbol{x}, t)=\omega_{p}$ the control function (2) will introduce compressible effects in (1). Therefore, before adding the control term to (1) one needs to project it on its solenoidal component.

The projection operation breaks the local positive definiteness of the control term, however, its global effect remains purely dissipative after averaging on the whole volume. Hence, the control term goes in addition to the normal dissipation produced by the kinematic viscosity. In this way, a second possible channel is opened where the energy, injected by the large-scale forcing, can be dissipated. The total energy balance equations becomes

$$
\frac{1}{2} \partial_{t}\left\langle\boldsymbol{u}^{2}\right\rangle=\nu\left\langle\Delta \boldsymbol{u}^{2}\right\rangle-\left\langle\boldsymbol{f}_{c} \cdot \boldsymbol{u}\right\rangle+\langle\boldsymbol{u} \cdot \mathbf{F}\rangle,
$$

where we have the total kinetic energy, $E=\frac{1}{2}\left\langle\boldsymbol{u}^{2}\right\rangle$, the viscous dissipation $\varepsilon_{\nu}=\nu\left\langle\Delta \boldsymbol{u}^{2}\right\rangle$, the dissipation induced by the control mechanism, $\varepsilon_{c}=\left\langle\boldsymbol{f}_{c} \cdot \boldsymbol{u}\right\rangle$, and the energy injection rate $\varepsilon_{f}=\langle\mathbf{F} \cdot \boldsymbol{u}\rangle$, and with $\langle\bullet\rangle$ we intend an average on the whole volume.

Numerical simulations. - In Fig. 1 we present two visualizations of a plane of the vorticity intensity in the stationary state for two simulations, one for the standard NSE (top panel) and one with the control term acting on the flow (bottom panel). The two planes in Fig. 1 are warped upwards depending on the vorticity values, in this way it is possible to see that the intense peaks developed by the NSE are pruned by the small-scale forcing in the controlled dynamics. From the figure it is qualitatively evident that vorticity is strongly depleted when the small-scale drag is acting, as expected. In the same figure, next to the vorticity planes, we show a $3 \mathrm{D}$ rendering of the contour regions where the vorticity value is above $20 \%$ of its maximum value, for the case of the uncontrolled NSE (top panel) and the contour regions where the vorticity value is above the forcing threshold, $p=0.2$, for the case of the controlled flow (bottom panel). From the volume rendering, we can appreciate that the control forcing tends to homogenize the spatial distribution of the intense vorticity events while they result more intermittently and localized when the dynamics is not controlled. One possible interpretation of this effect is that the control term is highly nonlinear, hence its dynamics can lead to a decorrelation in the time evolution of the Fourier phases at different scales and consequently to a depletion of intermittency. Indeed synchronization among

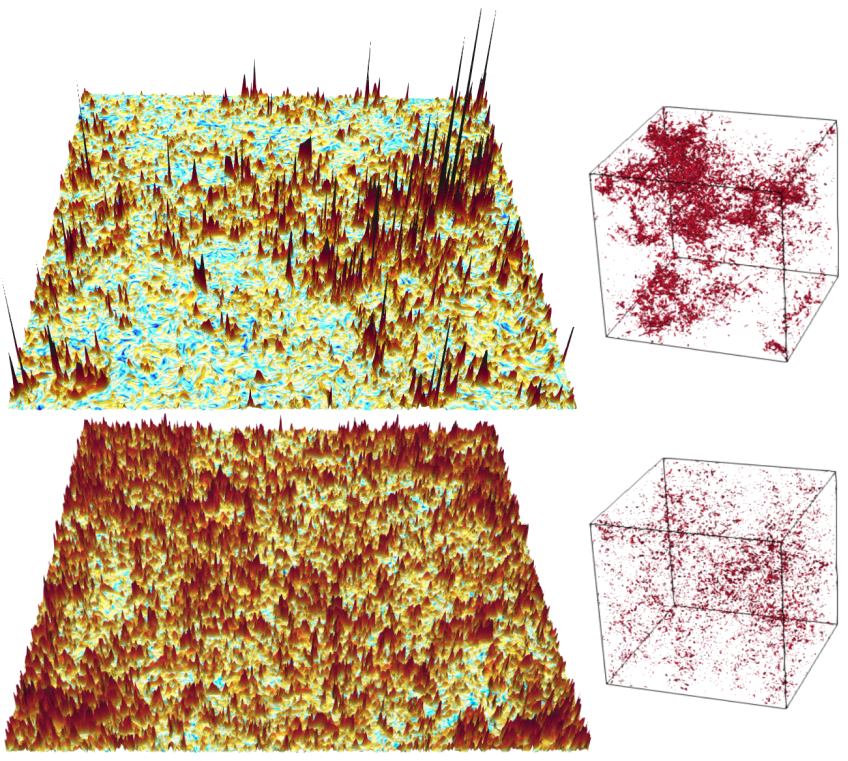

FIG. 1. (Top row) Left: vorticity amplitude in a 2D plane, from a simulation without control term $(\beta=0)$. Right: regions where the vorticity amplitude is above $20 \%$ of its maximum over the flow volume. (Bottom row) Left: visualization of enstrophy plane from a simulation with active control term. Both visualizations are obtained using the same range in the color axis. Right: regions where the vorticity amplitude is above the control forcing threshold $\omega_{p}=0.2 \omega_{\max }$. The control forcing amplitude is $\beta=5$. Both simulations are performed with $N=1024^{3}$ collocation points.

Fourier phases has been already observed to be key for the formation of high singular and intermittent structure in the Burgers equation [24,25] and it is believed to be important for Navier-Stokes equations too. It is also interesting to observe that the volume fraction where the forcing acts is very small, even though in those visualizations we are using

TABLE I. Control: indicates if the term (2) is applied (On) or not (Off); $N$ is the number of collocation points for each direction; $\beta$ is the amplitude of the control term; $p$ is the percentage of the maximum vorticity above which the control term is active $\omega_{p}=p \omega_{\max } ; \varepsilon_{f}$ is the mean energy input injected by the large-scale forcing; $\nu$ is the kinematic viscosity. The amplitude of the Ornstein-Uhlenbeck forcing is $f_{0}=0.16$ and $f_{0}=0.14$ for $N=256$ and $N=1024$, respectively; the correlation time is $\tau_{f}=0.6$ for $N=256$ and $\tau_{f}=0.23$ for $N=1024$. The forcing is active on the window $k_{f}=[0.5: 1.5]$ for resolution $N=256$ and on $k_{f}=[0.5: 2.5]$ for $N=1024$. The Kolmogorov scale is $\eta=\left(\nu^{3} / \varepsilon\right)^{1 / 4}$, where $\varepsilon$ is the dissipation rate. Resolution is kept at $\eta / d x \geq 0.7$.

\begin{tabular}{lccccc}
\hline \hline Control & $N$ & $\beta$ & $p$ & $\varepsilon_{f}$ & $\nu$ \\
\hline Off & 256 & $\ldots$ & $\ldots$ & 2.2 & $5.2 \times 10^{-3}$ \\
Off & 1024 & $\ldots$ & $\ldots$ & 5.5 & $8 \times 10^{-4}$ \\
On & 256 & {$[0.1 \div 50]$} & {$[0.1 \div 0.7]$} & 2.2 & $5.2 \times 10^{-3}$ \\
On & 1024 & 50 & {$[0.05 \div 0.6]$} & 5.5 & $8 \times 10^{-4}$ \\
\hline \hline
\end{tabular}


a broad threshold in terms of the vorticity values, $p=\omega_{p} / \omega_{\max }=0.2$.

To assess the statistical properties of Eq. (1) a set of direct numerical simulation have been performed at changing resolution and the control parameters, namely $\beta$ and $\omega_{p}$. We used a pseudospectral code with resolutions up to $1024^{3}$ collocation points in a triply periodic domain $\Omega$ of size $L=2 \pi$. A full $2 / 3$ dealiasing is implemented (see Table I for details). The homogeneous and isotropic external force, F, is defined via a second-order Ornstein-Uhlenbeck process [26]. All simulations where control is on, have been produced starting from a stationary configuration of the uncontrolled case $\beta=0$ and all statistical quantities are calculated after that a new stationary state is achieved.

In Fig. 2 we present the time average of the instantaneous energy spectra:

$$
E(k, t)=0.5 \sum_{k<|\boldsymbol{k}|<k+1}|\hat{\boldsymbol{u}}(\boldsymbol{k}, t)|^{2},
$$

which are almost independent of the control parameter, $p$. Only for the smallest value of $\omega_{p}$, with $p \sim 0.2$, we can notice a small energy depletion at large wave numbers. However, in all cases, the inertial range scaling properties are unchanged with the slope being very close to the Kolmogorov's prediction $k^{-5 / 3}$. In the inset of the same figure we show for the controlled simulation with $p=0.2$ and $\beta=5$, the balance of the energy flux produced by the nonlinear term, $\Pi_{n l}(k)$, by the viscous drag, $\Pi_{\nu}(k)$, and by the control forcing, $\Pi_{f_{c}}(k)$. In the stationary state we can write the Fourier space energy balance equation as:

$$
\Pi_{n l}(k)+\Pi_{f_{c}}(k)+\Pi_{\nu}(k)=\varepsilon_{f},
$$

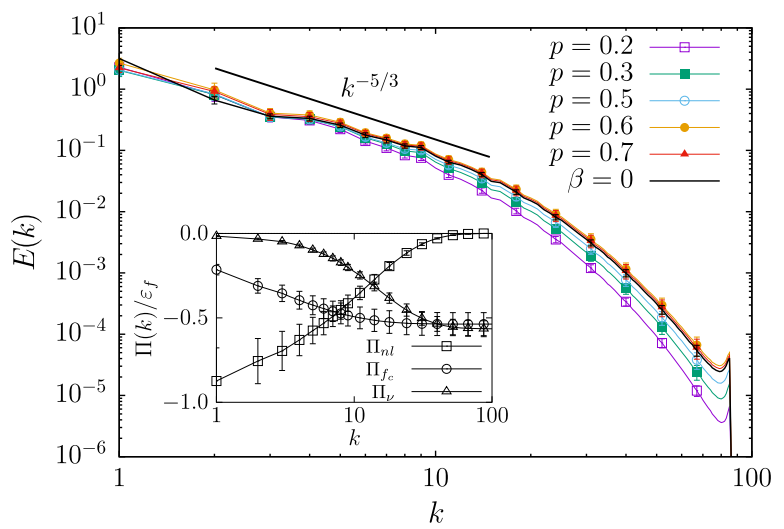

FIG. 2. Energy spectra averaged on time for different simulations with $N=256$ at changing the control threshold, $\omega_{p} / \omega_{\max }=p$, with a fixed amplitude, $\beta=5$. Notice that $\beta=$ 0 corresponds to the uncontrolled full NSE. Inset: energy fluxes contributions, nonlinear term $\left(\Pi_{n l}\right)$, control term $\left(\Pi_{f_{c}}\right)$, and viscous term $\left(\Pi_{\nu}\right)$ normalized to the mean energy input, $\varepsilon_{f}$. Here $p=0.2$ and $\beta=5$. where $\varepsilon_{f}$ is the large-scale energy input of the stochastic forcing. From the inset of Fig. 2 we can see that the control forcing is mainly active in the high wave numbers where its contribution equals the one from the viscous dissipation, while at small or intermediate wave numbers the nonlinear interactions remain the leading one.

Configuration space statistics.-In the following we analyze the statistics of the longitudinal velocity increments defined as $\delta_{r} u=[\boldsymbol{u}(\boldsymbol{x}+\boldsymbol{r})-\boldsymbol{u}(\boldsymbol{x})] \boldsymbol{r} / r$. In particular we are interested in the assessment of the effects produced by the control term on the intermittent properties of the NSE. To do that we study the scaling properties of the longitudinal structure functions (SF) defined as:

$$
S_{p}(r) \equiv\left\langle\left[\delta_{r} u\right]^{p}\right\rangle \sim r^{\zeta_{p}} .
$$

Intermittency is measured by the departure of the scaling exponents from the Kolmogorov 1941 prediction, $\zeta_{p}=$ $p / 3$ in the inertial range, $\eta<r<L_{0}$. In particular, any systematic nonlinear dependency on the order of the moment will induce a scale dependency in the flatness, defined by the dimensionless ratio among fourth and second order SF:

$$
F(r)=\frac{S_{4}(r)}{\left[S_{2}(r)\right]^{2}} .
$$

The flatness for the controlled turbulent flow at resolution $N=1024^{3}$ is presented in Fig. 3, for the case with $p=0.2$, compared with the uncontrolled case $\beta=0$ and with the uncontrolled case but with an a posteriori pruning of all events where $\omega>\omega_{p}$. The latter measurement is introduced in order to understand how much the dynamical pruning imposed by the evolution of Eq. (1) is different from a simple conditioning on small-vorticity events taken on the full uncontrolled NSE. It is important to underline that the a posteriori analysis is actually performing a conditional statistical averaging of the velocity increments and not a proper pruning of the velocity fields. In other words, in the a posteriori analysis, we skip in the computation of the structure functions those positions where the vorticity amplitude exceeds the threshold used in the dynamical control. A real a posteriori pruning protocol would have required to pad to zero the velocity inside the high vorticity regions, consequently introducing artificial discontinuities and thus higher values for the flatness. As one can see comparing the empty circles (full $\beta=0 \mathrm{NSE}$ ) with the empty squares (active control with $p=0.2$ ) the effects on the flatness are dramatic, with both a $100 \%$ reduction on the smallest scale and a decrease of the scaling slope in the inertial range. Similarly, by comparing the results with the a posteriori conditioning (empty triangles) we see that indeed it is crucial to have a dynamical control to deplete intermittency. In the Supplemental Material [27] we present an analysis of 


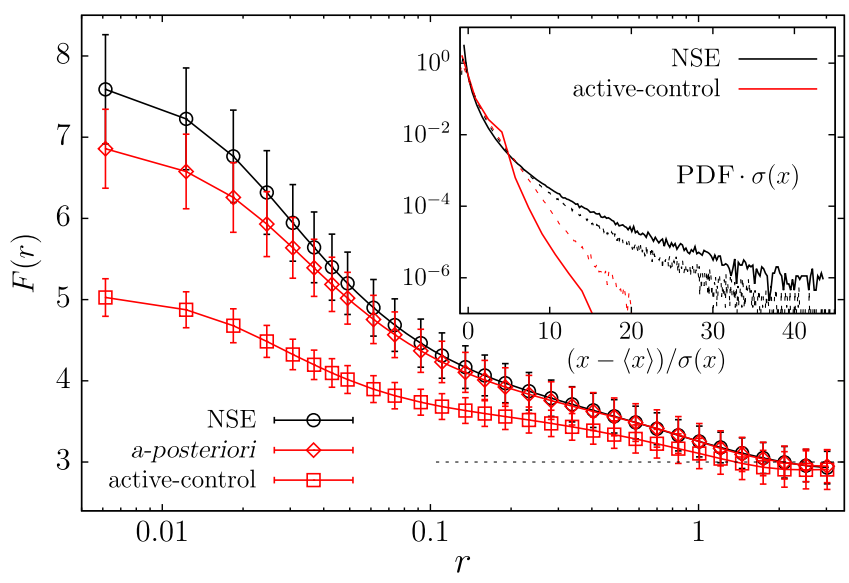

FIG. 3. Log-log plot of the flatness, $F(r)$, versus $r$ from 30 different time snapshots at resolution $N=1024^{3}$. Results without control terms, $\beta=0$ (NSE). Data with active control $\omega_{p} / \omega_{\max }=0.2, \beta=50$. The dashed line at $F(r)=3$ represents the expected value for a Gaussian distribution. We also show the flatness from a posteriori pruning. Errors are evaluated as the standard deviation from 30 configurations. Inset: comparison of the PDFs of enstrophy, $x=\Omega$, (solid lines) and shear intensity, $x=\mathcal{S}$ (dashed lines) measured from simulations of standard NSE (black lines) and from the system controlled with forcing threshold, $\omega_{p} / \omega_{\max }=0.2$ and amplitude $\beta=5$ (red lines).

the flatness at changing the control amplitude $\beta$ and the width of tanh function in Eq. (2). The flatness behaviour is qualitatively unchanged when varying the control parameters over a wide range of values, confirming that the intermittency reduction is not due to a particular choice of the control term. To our knowledge this is the first evidence that intermittency can be strongly depleted in a dynamical way with a dynamical criterion based on configurationspace filtering, at difference from what obtained by fractal pruning in [28-31]. In the inset of Fig. 3 we show the effects of the vorticity control point by point in the flow volume, by plotting the standardised probability density function (PDF) for the instantaneous and local enstrophy, $\Omega=|\nabla \times \boldsymbol{u}|^{2}$, and shear intensity, $\mathcal{S}=\sum_{i j}\left(\partial_{i} u_{j}+\partial_{j} u_{i}\right)^{2}$, for one case of active control, $p=0.2$, and compared with the no-control, $\beta=0$ case. There are two interesting things to remark. First, when the control is active, the far tails of the vorticity are markedly depleted, with almost a sharp cutoff at $\omega \sim \omega_{p}$, which is the clear signature that the control is able to deplete intense vorticity events and to not allow them to grow again during the evolution. This fact is also good news from a sort of min-max approach; it means that the amount of control needed is not too high, being very efficient in stopping the formation of strong vorticity. The second interesting point to remark is that the preferential depletion on vorticity is indeed changing the topological distribution of extreme events in the flow: from the standard case where they are mainly given by high vorticity where no control exists to the case where the extreme fluctuations (far right tails) are more dominated by strong shear events. The control term seems to introduce other subleading effects such as the formation of a small bump in the vorticity distribution; this is visible in the inset of Fig. 3 from the relative increase of the probability of measuring enstrophy values around the cutoff threshold of the control term. This small effect would require more work to be quantitatively clarified and it is most probably highly sensitive to the specific functional shape of the control forcing.

Drag reduction.-Going back to the observation of the mean quantities, it is interesting to estimate the effect of the smart forcing on the drag coefficient of the system. Indeed the new smart control allows the system to preferentially dissipate energy inside the vortical regions where it is active. It is important to remark that in the absence of boundaries the best way to identify a drag-reduction mechanism is to compare the energy injection rate (input) and the response of the flow via the total kinetic energy (output), a well-established procedure, as already suggested in [32-34]. Hence to quantify the smart-control effect we go back to the balance (3) and split the total drag, $d_{\text {tot }}$, in two contributions, $d_{\nu}$ and $d_{c}$, as follows:

$$
d_{\mathrm{tot}}=d_{\nu}+d_{c}, \quad d_{\nu}=\frac{\varepsilon_{\nu} L_{0}}{u_{\mathrm{rms}}^{3}} ; \quad d_{c}=\frac{\varepsilon_{c} L_{0}}{u_{\mathrm{rms}}^{3}} .
$$

In Fig. 4 we show the mean drag coefficients as a function of the vorticity threshold $\omega_{p}=p \omega_{\max }$ for the simulations with $N=256^{3}$ collocation points and with a moderate control amplitude, $\beta=5$. Figure 4 shows that the drag contribution coming from the control term is negligible up

$V$

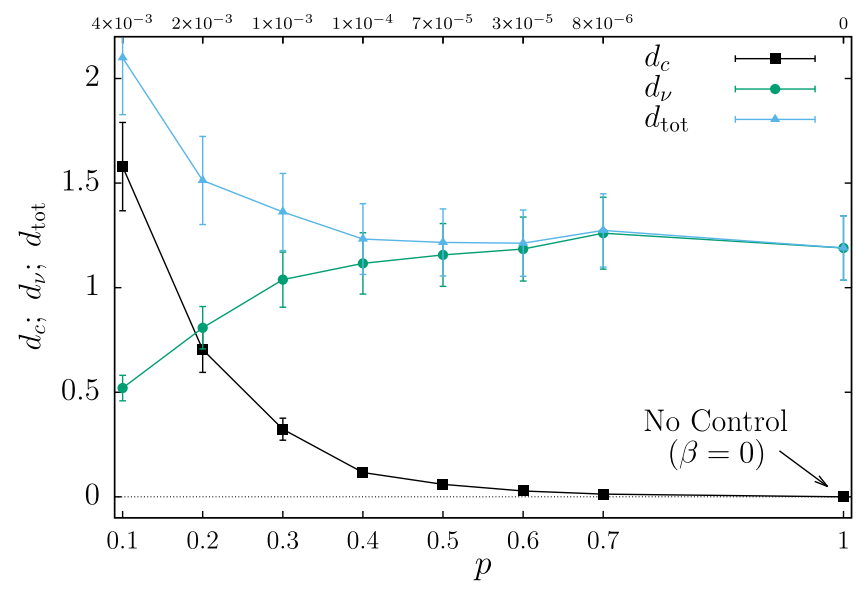

FIG. 4. Drag coefficient for the viscous dissipation $d_{\nu}$ (green line), the control forcing $d_{c}$ (black line), and their sum $d_{\text {tot }}$ (cyan line). Results are shown as a function of the threshold, $\omega_{p} / \omega_{\max }=p$, and the volume fraction, $V$, where the control forcing is acting (upper scale). Here the resolution is $N=256^{3}$ and the control forcing amplitude is $\beta=5$. Errors are evaluated as the standard deviation of the temporal fluctuations. 
to a threshold $p \sim 0.6$, instead, moving towards lower thresholds, the dissipation produced by the small-scale term increases and, around $p=0.2$, the kinematic viscosity and the control dissipations become of the same order. Moving further the threshold towards lower vorticity values the control term becomes the leading contribution responsible for the energy dissipation. In this way, a drag enhancement is observed for the smaller threshold value and the overall drag coefficient is increased almost by a factor of 2 compared to the free NSE at $p=1$.

Conclusions. - We have presented a first implementation of a smart small-scale control scheme for turbulent flows, based on preferentially dumping high vorticity regions. In this Letter, we have shown that the extra drag exerted on the vortex filaments produce a strong reduction on configuration-based intermittency, with the depletion of fat tails and rare events in the vorticity field. The topological relative weight of rotational and extensional regions is also affected abruptly. The overall dumping of vortex filaments leads to a sort of drag increase. This study opens the way to explore other control Lagrangian mechanism, e.g., based on the heavy-light particles preferential concentration and/or other smart particles that can be self-activated or activated by external control fields, as for the case of magnetic objects. Together with the study of possible real Lagrangian control mechanisms, it would be interesting to study the effects of the new control approach when applied to turbulent flows in different and more realistic conditions such as in rotating, stratified, or shear flows, where the turbulent dynamics is known to develop different energy cascades. In some cases, vorticity is intense even at large scales, which would imply the application of a control mechanisms that is correlated on scales comparable with the domain size. Optimization of the particles' properties to track a specific flow region can also be attempted in order to enhance or deplete only specific fluctuations [35-39].

\section{*f.toschi@tue.nl}

[1] U. Frisch, Turbulence: The Legacy of A. N. Kolmogorov (Cambridge University Press, Cambridge, England, 1995).

[2] S. B. Pope, Turbulent Flows (Cambridge University Press, Cambridge, 2000)

[3] R. Benzi, L. Biferale, R. Fisher, D. Lamb, and F. Toschi, J. Fluid Mech. 653, 221 (2010).

[4] P. K. Yeung, X. M. Zhai, and K. R. Sreenivasan, Proc. Natl. Acad. Sci. U.S.A. 112, 12633 (2015).

[5] K. P. Iyer, K. R. Sreenivasan, and P. K. Yeung, Phys. Rev. E 95, 021101(R) (2017).

[6] M. Sinhuber, G. P. Bewley, and E. Bodenschatz, Phys. Rev. Lett. 119, 134502 (2017).

[7] J. Bec, L. Biferale, M. Cencini, A. Lanotte, S. Musacchio, and F. Toschi, Phys. Rev. Lett. 98, 084502 (2007).

[8] F. Toschi and E. Bodenschatz, Annu. Rev. Fluid Mech. 41, 375 (2009).

[9] E. Calzavarini, R. Volk, M. Bourgoin, E. Lvque, J. F. Pinton, and F. Toschi, J. Fluid Mech. 630, 179 (2009).
[10] N. M. Qureshi, U. Arrieta, C. Baudet, A. Cartellier, Y. Gagne, and M. Bourgoin, Eur. Phys. J. B 66, 531 (2008).

[11] J. M. Mercado, D. C. Gomez, D. Van Gils, C. Sun, and D. Lohse, J. Fluid Mech. 650, 287 (2010).

[12] M. Gibert, H. Xu, and E. Bodenschatz, J. Fluid Mech. 698, 160 (2012).

[13] K. Gustavsson and B. Mehlig, J. Fluid Mech. 65, 1 (2016).

[14] V. Mathai, E. Calzavarini, J. Brons, C. Sun, and D. Lohse, Phys. Rev. Lett. 117, 024501 (2016).

[15] R. Stanway, Mater. Sci. Technol. 20, 931 (2004).

[16] E. Falcon, J. C. Bacri, and C. Laroche, Phys. Rev. Fluids 2, 102601 (2017).

[17] Y. Abe, M. Kawaji, and T. Watanabe, Exp. Therm. Fluid. Sci. 26, 817 (2002).

[18] M. Hauptmann, H. Struyf, S. De Gendt, C. Glorieux, and S. Brems, J. Appl. Phys. 113, 184902 (2013).

[19] M. Hauptmann, H. Struyf, S. De Gendt, C. Glorieux, and S. Brems, ECS J. Solid State Sci. 3, N3032 (2014).

[20] H. W. Huang, F. E. Uslu, P. Katsamba, E. Lauga, M. S. Sakar, and B. J. Nelson, Sci. Adv. 5, eaau1532 (2019).

[21] J. L. Lumley, J. Polym. Sci. 7, 263 (1973).

[22] C. M. White and M. G. Mungal, Annu. Rev. Fluid Mech. 40, 235 (2008).

[23] P. Fischer and A. Ghosh, Nanoscale 3, 557 (2011).

[24] M. Buzzicotti, B. Murray, L. Biferale, and M. D. Bustamante, Eur. Phys. J. E 39, 34 (2016).

[25] B. Murray and M. D. Bustamante, J. Fluid Mech. 850, 624 (2016).

[26] L. Biferale, F. Bonaccorso, I. M. Mazzitelli, M. A. T. van Hinsberg, A. S. Lanotte, S. Musacchio, P. Perlekar, and F. Toschi, Phys. Rev. X 6, 041036 (2016).

[27] See the Supplemental Material at http://link.aps.org/ supplemental/10.1103/PhysRevLett.124.084504 for further quantitative results on the effects of changing the free parameters in the forcing model.

[28] A. S. Lanotte, R. Benzi, S. K. Malapaka, F. Toschi, and L. Biferale, Phys. Rev. Lett. 115, 264502 (2015).

[29] M. Buzzicotti, L. Biferale, U. Frisch, and S. S. Ray, Phys. Rev. E 93, 033109 (2016).

[30] A. S. Lanotte, S. K. Malapaka, and L. Biferale, Eur. Phys. J. E 39, 49 (2016).

[31] M. Buzzicotti, A. Bhatnagar, L. Biferale, A. S. Lanotte, and S. S. Ray, New. J. Phys. 18, 113047 (2016).

[32] E. De Angelis, C. M. Casciola, R. Benzi, and R. Piva, J. Fluid Mech. 531, 1 (2005).

[33] R. Benzi, E. De Angelis, R. Govindarajan, and I. Procaccia, Phys. Rev. E 68, 016308 (2003).

[34] G. Boffetta, A. Celani, and A. Mazzino, Phys. Rev. E 71, 036307 (2005).

[35] G. Reddy, A. Celani, T. J. Sejnowski, and M. Vergassola, Proc. Natl. Acad. Sci. U.S.A. 113, E4877 (2016).

[36] S. Colabrese, K. Gustavsson, A. Celani, and L. Biferale, Phys. Rev. Lett. 118, 158004 (2017).

[37] S. Colabrese, K. Gustavsson, A. Celani, and L. Biferale, Phys. Rev. Fluids 3, 084301 (2018).

[38] A. Waldock, C. Greatwood, F. Salama, and T. Richardson, Journal of Intelligent and Robotic Systems: Theory and Applications 92, 685 (2018).

[39] G. Novati, L. Mahadevan, and P. Koumoutsakos, Phys. Rev. Fluids 4, 093902 (2019). 